\title{
Efficacy of Two-Stage Surgery for Spinal Cord Ependymomas
}

\author{
Soichiro Takamiya ${ }^{1}$, Toshitaka Seki ${ }^{1}$, Kazuyoshi Yamazaki ${ }^{1}$, Shunsuke Yano ${ }^{2}$, Kazutoshi Hida ${ }^{2}$ \\ ${ }^{1}$ Department of Neurosurgery, Faculty of Medicine and Graduate School of Medicine, Hokkaido University, Sapporo, Japan \\ ${ }^{2}$ Department of Neurosurgery, Sapporo Azabu Neurosurgical Hospital, Sapporo, Japan
}

Study Design: Retrospective cohort study.

Purpose: This study aimed to elucidate cases for which staged surgeries are effective by a retrospective review of previous operative cases of spinal ependymomas.

Overview of Literature: Patients with spinal ependymomas are expected to have a good prognosis following total resection. However, forcible dissection of spinal ependymomas will lead to neurological deterioration. Moreover, resection is sometimes difficult when the tumor is large. We have performed two-stage surgeries for large spinal ependymomas, but the indication of staged surgery is unclear.

Methods: We retrospectively reviewed patients diagnosed with spinal ependymomas who underwent tumor resection in our institution. We obtained data regarding patients' clinical characteristics, tumoral radiological characteristics, and surgical factors and compared them to clear prognostic factors. Two-stage surgery was performed in 11 patients (36.7\%), and single surgery was performed in 19 patients $(63.3 \%)$.

Results: Thirty patients were included in the analyses and divided into two groups: single surgery and two-stage surgery groups. In the single surgery group, high tumor-cord ratio (TCR) and intraoperative motor evoked potential (MEP) reduction were significantly correlated with unfavorable outcomes, which were defined as deterioration of the modified McCormick scale grades 2 months and 1 year postoperatively. Alternatively, these factors were not significantly correlated with postoperative unfavorable outcomes in the two-stage surgery group. Receiver operating characteristic curves indicated that TCR of 0.866 yielded $85.7 \%$ sensitivity and $83.3 \%$ specificity 2 months postoperatively.

Conclusions: The results suggested that high TCR might be an indication of two-stage surgery and that its cutoff value is 0.866 . Moreover, switching from single surgery to two-stage surgery may prevent postoperative neurological deterioration when intraoperative MEP is decreasing.

Keywords: Spinal cord; Ependymoma; Two-stage surgery

\section{Introduction}

Spinal ependymomas are benign spinal intramedullary tumors with a well-circumscribed plane between the tumor and spinal cord [1], and a good prognosis can be expected after gross total resection (GTR) [1-4]. Alternatively, rough dissection could lead to neurological deterioration; therefore, careful dissection is required [5]. However,

Received Apr 13, 2021; Revised Apr 27, 2021; Accepted May 11, 2021

Corresponding author: Soichiro Takamiya

Department of Neurosurgery, Faculty of Medicine and Graduate School of Medicine, Hokkaido University, North 15, West 7, Kita-ku, Sapporo, Hokkaido, 060-8638, Japan

Tel: +81-11-706-5987, Fax: +81-11-708-7737, E-mail: soichiro.tkmy@med.hokudai.ac.jp 
when the tumor is large, careful resection of the tumor is difficult. Several surgeons have reported that two-stage surgery for spinal intramedullary tumors is a neuroprotective method [6-11], which can be divided into three parts: (1) first surgery: laminoplasty and/or laminectomy, myelotomy, and dorsal tumor dissection; (2) between the two surgeries: tumor resection by cerebrospinal fluid and "tumor delivery" by intraparenchymal pressure; and (3) second surgery: ventral tumor dissection. Large spinal ependymomas are a good indication of two-stage surgery. We have been performing two-stage surgeries for large spinal ependymomas since 2000. Nevertheless, we have not had a precise criterion in determining which case for which two-stage surgery is effective. Thus, this study elucidates the cases for which two-stage surgery is effective by retrospectively reviewing previous operative cases of spinal ependymomas.

\section{Materials and Methods}

\section{Patient selection}

We retrospectively reviewed patients diagnosed with spinal ependymoma (World Health Organization [WHO] grade II) who underwent single or two-stage surgery at Hokkaido University Hospital from 2005 to 2018. Patients with myxopapillary ependymomas (WHO grade I) and anaplastic ependymomas (WHO grade III) were excluded.

This study was conducted in compliance with the principles of the Declaration of Helsinki. Informed consent was obtained in the form of opting in on the website, and those who rejected were excluded. This study was approved by the Institutional Review Board of Hokkaido University Hospital (approval no., 019-0387).

\section{Surgical procedures}

\section{1) Single surgery}

Patients were placed in a neutral prone position. A midline linear skin incision was made, and double open-door laminoplasty and/or laminectomy was performed to adequately expose the dura to remove the tumor. After dural incision, myelotomy was performed through the posterior median sulcus. When the tumor was encountered, it was dissected gently along the tumor-spinal cord interface. When the tumor was dissected in the whole circumference and length, it was removed. Then, the pial edges were roughly sutured, and the dura mater was primarily closed with the arachnoid membrane.

\section{2) Two-stage surgery}

In the first surgery, procedures until myelotomy were the same as those of single surgery (Fig. 1A). When the tumor was encountered, it was dissected gently along the tumorspinal cord interface. The dissection remained on the dorsal surface of the tumor, and duraplasty using a GORETEX membrane (W. L. Gore \& Associates Inc., Tokyo, Japan) was performed (Fig. 1B).

After 1 or 2 weeks, the second surgery was performed. In all cases, the tumor extruded from the surface of the spinal cord (Fig. 1C). The tumor dissection was continued to the ventral surface of the tumor, which was then removed (Fig. 1D). The closure procedures were similar to those of single surgery.

\section{Data collection}

Data associated with patient, tumor, and surgical factors were collected as follows. Each parameter was reviewed in all patients, single surgery group, and two-stage surgery group.

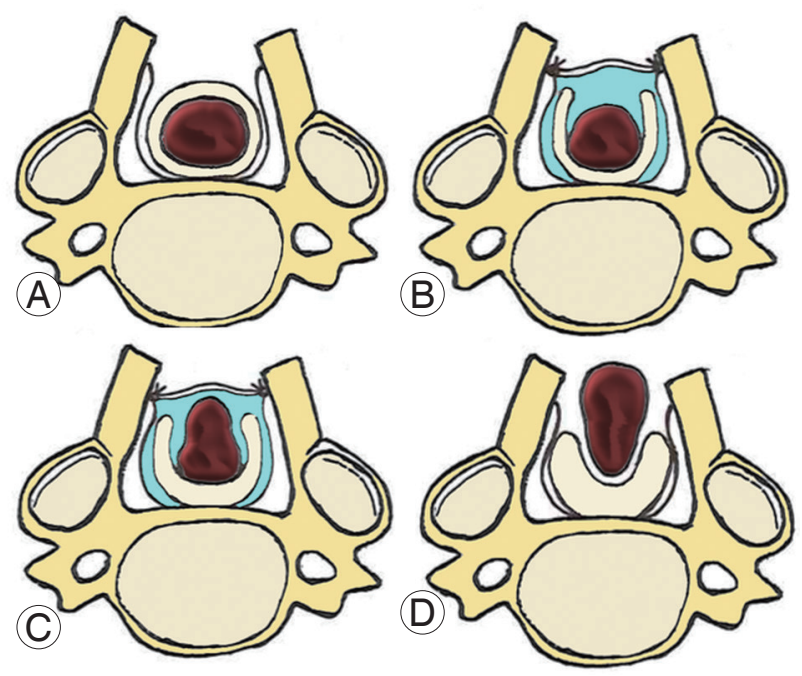

Fig. 1. Schema of two-stage surgery. (A) After laminoplasty and/or laminectomy, the dura matter is opened and the spinal cord is exposed. (B) Myelotomy is performed via the posterior median sulcus, and the tumor is dissected along the tumor-spinal cord interface. The dissection remains on the dorsal surface of the tumor, and duraplasty with GORE-TEX membrane is performed. (C) After the first surgery, the dorsal part of the tumor extrudes from the spinal cord. (D) In the second surgery, the ventral surface of the tumor is dissected. 


\section{1) Patient factors}

Data on age, sex, and duration of symptoms were reviewed. Moreover, preoperative and postoperative 2-month and postoperative 1-year neurological functions of the patients were evaluated using the modified McCormick scale (MMS) using the following grading system [12]: grade I, neurological normal or mild focal deficit (normal gait); grade II, sensorimotor deficit affecting functions of the involved limb (having gait difficulty but independent); grade III, more severe neurological deficit (requiring cane/brace for ambulation); and grade IV, severe deficit (requiring wheelchair or bilateral cane/brace). A favorable outcome is defined as MMS grades that are improving or stable postoperatively. Alternatively, an unfavorable outcome is defined as MMS grades that are worsening postoperatively.

\section{2) Tumor factors}

Tumors were evaluated using gadolinium-enhanced T1weighted magnetic resonance images (MRIs).

Tumor locations were classified into the following eight cord levels: medulla (M), upper cervical (uC), lower cervical (lC), upper thoracic (uT), middle thoracic (mT), lower thoracic (lT), lumbar (L), and conus (Co). Considering that discrepancies exist between the vertebral and cord levels (myelomeres), the aforementioned eight cord levels were defined as the following vertebral levels: $\mathrm{M}$, above C1; uC, C1-C4; 1C, C4-C7; uT, C7-T3; mT, T3-T7; 1T, T7-T10; L, T10-T12; and Co, below T12. Tumor locations were permitted to have overlapping distributions. For example, if the tumor exists from $\mathrm{C} 2$ to $\mathrm{T} 1$ vertebral levels, it is expressed as $\mathrm{uC}, \mathrm{C}$, and $\mathrm{uT}$ in this study.

The tumor lengths were measured between the cranial and caudal ends of the gadolinium-enhanced lesions in the sagittal view (Fig. 2A). When the tumor was curve shaped, lengths were measured from the middle point to both ends of the tumor, and both values were summed up to determine the final tumor length (Fig. 2B).

Moreover, the tumor-cord ratio (TCR) was measured in the cross-sectional view. TCR was defined as the ratio of the anteroposterior (AP) diameter of the tumor to the AP diameter of the spinal cord in the section where the AP diameter of the tumor was at maximum (Fig. 2C). Moreover, the existence of tumor cysts was reviewed.

\section{3) Surgical factors}

Whether the intraoperative motor evoked potential (MEP) decreased was evaluated.
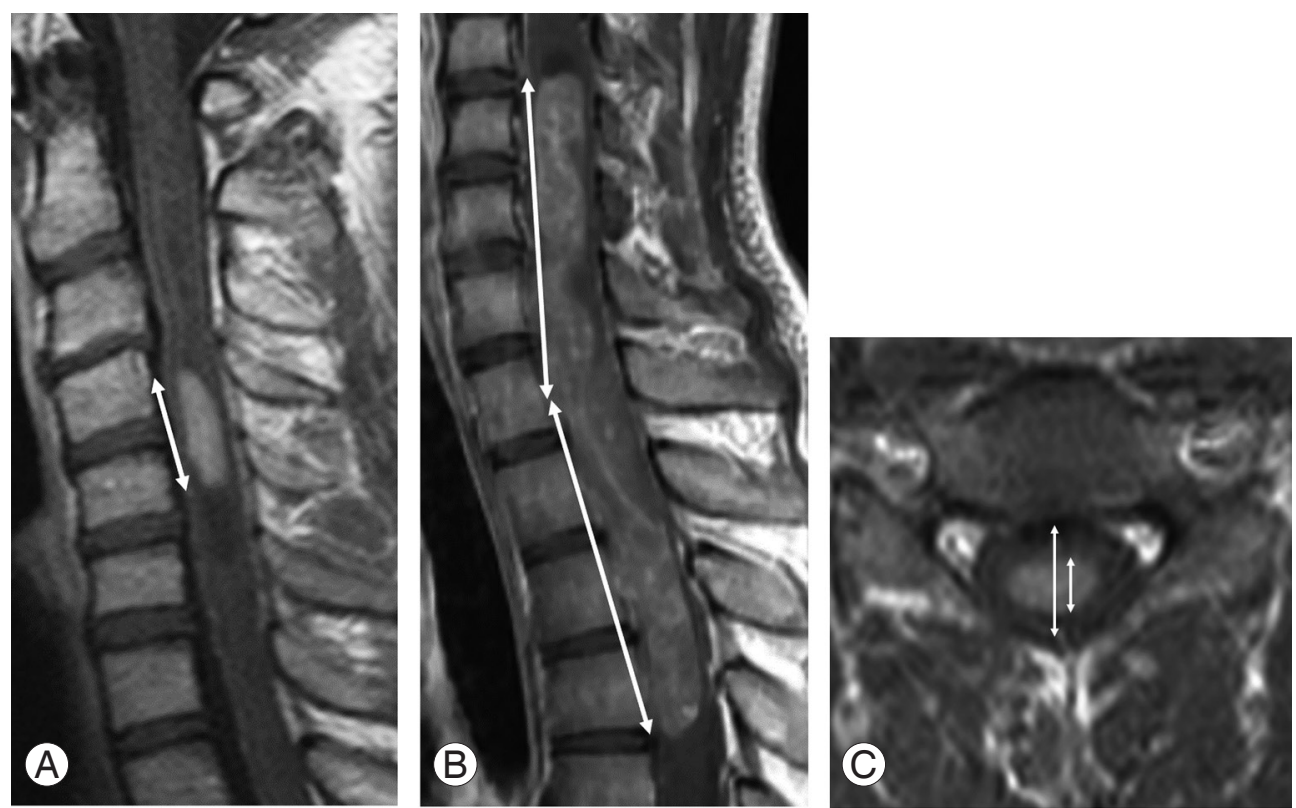

Fig. 2. Measurement of tumoral parameters on magnetic resonance imaging. (A) Tumor length was measured between the cranial and caudal ends of gadolinium-enhanced lesions on the sagittal view of gadolinium-enhanced T1-weighted images (arrow). (B) When the tumor length could not be measured linearly, the sum of the upper and lower parts of the tumor, which were divided by the midpoint, was obtained (arrow). (C) The tumor-cord ratio was measured as the ratio of the anteroposterior (AP) diameter of the tumor to the AP diameter of the spinal cord on the cross-sectional view (arrow). 


\section{Statistical analyses}

The data were analyzed using JMP Pro ver. 14.0.0 (SAS Institute Inc., Cary, NC, USA). Comparisons between groups were performed using Wilcoxon rank sum test and chi-square test (or Fisher's exact test), and receiver operating characteristic (ROC) curves were made. All $p$-values $<0.05$ were used to denote statistical significance.

\section{Results}

\section{Summary of whole data}

Thirty patients with spinal ependymomas were included in the analysis. Data regarding the patients' clinical characteristics, tumoral radiological characteristics, and surgical factors are presented in Table 1.

The median age of patients was 39.5 years (range, 18-73 years). Among 30 patients, 18 (60.0\%) were men, and 12
(40.0\%) were women. The median symptom duration was 12.5 months (range, 1-97 months). Regarding the preoperative neurological status based on the MMS, 5 (16.7\%) were grade I, $24(80.0 \%)$ were grade II, and 1 (3.3\%) was grade IV. With respect to postoperative 2 -month and 1-year neurological status, 7 (23.3\%) and $9(30.0 \%)$ were grade I, $10(33.3 \%)$ and $14(46.7 \%)$ were grade II, 11 (36.7\%) and $6(20.0 \%)$ were grade III, and $2(6.7 \%)$ and $1(3.3 \%)$ were grade IV, respectively. Favorable outcomes were achieved in 18 patients (60.0\%) 2 months postoperatively and 23 patients $(76.7 \%) 1$ year postoperatively.

In terms of tumor location, $2(6.7 \%)$ were located in M, 13 (43.3\%) in uC, 17 (56.7\%) in lC, 8 (26.7\%) in uT, $4(13.3 \%)$ in $\mathrm{mT}, 3(10.0 \%)$ in $\mathrm{TT}$, and $3(10.0 \%)$ in L. No tumor was located in Co. The median \pm standard deviation (SD) tumor length was $43.2 \pm 6.2 \mathrm{~mm}$, and the median $\pm \mathrm{SD}$ TCR was $0.843 \pm 0.157$. Tumor cysts were concomitant with tumors in 26 patients. Half of the patients had decreased intraoperative MEP.

Table 1. Data of the patients' clinical characteristics, tumoral radiological characteristics, and surgical factors

\begin{tabular}{|c|c|c|c|c|}
\hline Characteristic & Total $(n=30)$ & Single $(n=19)$ & Two-stage ( $\mathrm{n}=11)$ & $p$-value \\
\hline \multicolumn{5}{|l|}{ Patients' clinical characteristics } \\
\hline Age (yr) & $39.5(18-73)$ & $45(26-73)$ & $31(18-56)$ & 0.0118 \\
\hline Sex & & & & 0.0086 \\
\hline Male & 60.0 & 78.9 & 27.3 & \\
\hline Female & 40.0 & 21.1 & 72.7 & \\
\hline Symptom duration (mo) & $12.5(1-97)$ & $14(2-97)$ & $11(1-33)$ & NS \\
\hline \multicolumn{5}{|l|}{ Tumoral radiological characteristics } \\
\hline \multicolumn{5}{|c|}{ Lesion level (overlapping distribution) } \\
\hline Medulla & 6.7 & 0 & 18.2 & NS \\
\hline Upper cervical & 43.4 & 31.6 & 63.6 & NS \\
\hline Lower cervical & 56.7 & 57.9 & 54.5 & NS \\
\hline Upper thoracic & 26.7 & 21.1 & 36.4 & NS \\
\hline Middle thoracic & 13.3 & 15.8 & 9.1 & NS \\
\hline Lower thoracic & 10.0 & 10.5 & 9.1 & NS \\
\hline Lumbar & 10.0 & 10.5 & 9.1 & NS \\
\hline Conus & 0 & 0 & 0 & NS \\
\hline Lesion length (mm) & $43.2(12.1-146.2)$ & $42.8(12.1-146.2)$ & $54.1(15.7-143.4)$ & NS \\
\hline Tumor-cord ratio & $0.843(0.367-0.96)$ & $0.82(0.367-0.96)$ & $0.856(0.476-0.949)$ & NS \\
\hline Tumor cyst & 86.7 & 84.2 & 90.9 & NS \\
\hline \multicolumn{5}{|l|}{ Surgical factors } \\
\hline Motor evoked potential reduction & 50.0 & 31.6 & 81.8 & 0.0209 \\
\hline
\end{tabular}

Values are presented as median (range) or \%.

NS, not significant. 
Two-stage surgery was performed in 11 patients (36.7\%), and single surgery was performed in 19 patients (63.3\%). In the comparison of two surgical methods, patients were younger and female-dominated in the twostage surgery group ( $p=0.0118$ and 0.0086 , respectively). Moreover, the two-stage surgery group had more patients with MEP reduction ( $p=0.0118$ ). However, other factors, including tumor length and TCR, were not significantly different.

\section{Comparison of favorable/unfavorable outcome rates in each factor}

Favorable outcome rates 2 months and 1 year postoperatively were evaluated in each factor as shown above. In each factor, the patients were divided into two groups, and favorable outcome rates were compared between the two groups. The factors categorized into an interval or ratio scale, such as the patient's age and tumor length, were divided using median values. These analyses were performed against (1) all patients, (2) patients who under- went single surgery, and (3) patients who underwent twostage surgery.

\section{1) All patients}

No significant differences in patient factors were found. Regarding tumor factors, patients with $\mathrm{uC}$ lesions were significantly more likely to have favorable outcomes 1 year postoperatively ( $p=0.0104$ ), and those with $\mathrm{mT}$ lesions were significantly more likely to have unfavorable outcomes 1 year postoperatively $(p=0.0307)$. Moreover, patients with high TCR were likely to have unfavorable outcomes 1 year postoperatively ( $p=0.0801$ ); however, tumor length and existence of tumor cysts were not significantly correlated with postoperative neurological status.

Regarding surgical factors, the number of surgeries (single or two-stage) was not correlated with favorable outcomes. Intraoperative MEP reduction was statistically significantly correlated with unfavorable outcomes 2 months postoperatively ( $p=0.0078$ ), and patients with reduced intraoperative MEP were likely to have unfavorable outcomes 1 year postoperatively $(p=0.0801)$.

Table 2. Comparison of favorable outcome rates in the single surgery group

\begin{tabular}{|c|c|c|c|c|c|}
\hline \multirow{2}{*}{ Variable } & \multirow{2}{*}{ Category } & \multicolumn{2}{|c|}{2 Months after surgery } & \multicolumn{2}{|c|}{1 Year after surgery } \\
\hline & & Favorable outcome $(\%)$ & $p$-value & Favorable outcome (\%) & $p$-value \\
\hline \multicolumn{6}{|l|}{ Patient factors } \\
\hline Age (yr) & $\leq 40 />40$ & $71.4 / 58.3$ & NS & $85.7 / 66.7$ & NS \\
\hline Sex & Male/female & $60.0 / 75.0$ & NS & $73.3 / 75.0$ & NS \\
\hline Duration (mo) & $\leq 12.5 />12.5$ & $66.7 / 60.0$ & NS & $77.8 / 70.0$ & NS \\
\hline \multicolumn{6}{|l|}{ Tumor factors } \\
\hline \multicolumn{6}{|l|}{ Level } \\
\hline Medulla & $-/+$ & $-/-$ & NS & $-/-$ & NS \\
\hline Upper cervical & $-/+$ & $53.9 / 83.3$ & NS & $61.5 / 100.0$ & NS \\
\hline Lower cervical & $-/+$ & $50.0 / 72.7$ & NS & $75.0 / 72.7$ & NS \\
\hline Upper thoracic & $-1+$ & $66.7 / 50.0$ & NS & $73.3 / 75.0$ & NS \\
\hline Middle thoracic & $-/+$ & $68.8 / 33.3$ & NS & $81.3 / 33.3$ & NS \\
\hline Lower thoracic & $-/+$ & $64.7 / 50.0$ & NS & $76.5 / 50.0$ & NS \\
\hline Lumbar & $-/+$ & $64.7 / 50.0$ & NS & $76.5 / 50.0$ & NS \\
\hline Lesion length (mm) & $\leq 43.2 />43.2$ & $70.0 / 55.6$ & NS & $70.0 / 77.8$ & NS \\
\hline Tumor-cord ratio & $\leq 0.843 />0.843$ & $90.0 / 33.3$ & 0.0198 & $100.0 / 44.4$ & 0.0108 \\
\hline Tumor cyst & $-/+$ & $100.0 / 56.3$ & NS & $100.0 / 68.8$ & NS \\
\hline \multicolumn{6}{|l|}{ Surgical factors } \\
\hline Motor evoked potential reduction & $-/+$ & $84.6 / 16.7$ & 0.0095 & $92.3 / 33.3$ & 0.0173 \\
\hline
\end{tabular}

NS, not significant. 
2) Patients who underwent single surgery (Table 2)

Among patient and tumor factors, only high TCR was significantly correlated with unfavorable outcomes 2 months and 1 year postoperatively $(p=0.0198$ and $p=0.0108$, respectively). Furthermore, a statistically significant correlation between intraoperative MEP reduction and unfavorable outcomes was found both 2 months and 1 year postoperatively ( $p=0.0095$ and $p=0.0173$, respectively).

3) Patients who underwent two-stage surgery (Table 3)

Only patients with a short symptom duration tended to have favorable outcomes 2 months postoperatively ( $p=0.0801)$; however, other factors, including TCR and MEP reduction, were not significantly correlated with postoperative neurological deficits.

Moreover, two surgical methods were compared among patients with higher TCR defined as more than the median value (>0.843). Then, two-stage surgery achieved more favorable outcomes both 2 months and 1 year postoperatively (66.7\% and $83.3 \%$, respectively) compared with single surgery (33.3\% and $44.4 \%$, respectively) although they were not statistically significant $(p=0.231$ and 0.168 , respectively).

\section{Cutoff value of tumor-cord ratio to perform two- stage surgery}

An ROC curve was produced by comparing high and low TCRs in the single surgery group to determine the definite cutoff value for unfavorable outcomes. The areas under the curve 2 months and 1 year postoperatively were 0.869 and 1.000 , respectively. The results indicated that TCR of 0.866 yielded $85.7 \%$ sensitivity and $83.3 \%$ specificity 2 months postoperatively, and TCR of 0.909 yielded $100 \%$ sensitivity and $100 \%$ specificity 1 year postoperatively. Therefore, the results suggest that TCRs $>0.866$ are a good indication for two-stage surgery, and single surgery could lead to neurological deterioration.

\section{Discussion}

In patients with spinal ependymomas, long-term survival

Table 3. Comparison of favorable outcome rates in the two-stage surgery group

\begin{tabular}{|c|c|c|c|c|c|}
\hline \multirow{2}{*}{ Variable } & \multirow{2}{*}{ Category } & \multicolumn{2}{|c|}{2 Months after surgery } & \multicolumn{2}{|c|}{1 Year after surgery } \\
\hline & & Favorable outcome $(\%)$ & $p$-value & Favorable outcome $(\%)$ & $p$-value \\
\hline \multicolumn{6}{|l|}{ Patient factors } \\
\hline Age (yr) & $\leq 40 />40$ & $55.6 / 50.0$ & NS & $77.8 / 100.0$ & NS \\
\hline Sex & Male/female & $66.7 / 50.0$ & NS & $100.0 / 75.0$ & NS \\
\hline Duration (mo) & $\leq 12.5 />12.5$ & $83.3 / 20.0$ & 0.0801 & 100.0/60.0 & NS \\
\hline \multicolumn{6}{|l|}{ Tumor factors } \\
\hline \multicolumn{6}{|l|}{ Level } \\
\hline Medulla & $-/+$ & $55.6 / 50.0$ & NS & $77.8 / 100.0$ & NS \\
\hline Upper cervical & $-/+$ & $50.0 / 57.1$ & NS & $50.0 / 100.0$ & NS \\
\hline Lower cervical & $-/+$ & $60.0 / 50.0$ & NS & $100.0 / 66.7$ & NS \\
\hline Upper thoracic & $-/+$ & $57.1 / 50.0$ & NS & $100.0 / 50.0$ & NS \\
\hline Middle thoracic & $-/+$ & $60.0 / 0.0$ & NS & $90.0 / 0.0$ & NS \\
\hline Lower thoracic & $-/+$ & $50.0 / 100.0$ & NS & $80.0 / 100.0$ & NS \\
\hline Lumbar & $-/+$ & $50.0 / 100.0$ & NS & $80.0 / 100.0$ & NS \\
\hline Lesion length (mm) & $\leq 43.2 />43.2$ & $60.0 / 50.0$ & NS & $100.0 / 66.7$ & NS \\
\hline Tumor-cord ratio & $\leq 0.843 />0.843$ & $40.0 / 66.7$ & NS & $80.0 / 83.3$ & NS \\
\hline Tumor cyst & $-1+$ & $100.0 / 50.0$ & NS & $100.0 / 80.0$ & NS \\
\hline \multicolumn{6}{|l|}{ Surgical factors } \\
\hline Motor evoked potential reduction & $-/+$ & $100.0 / 44.4$ & NS & $100.0 / 77.8$ & NS \\
\hline
\end{tabular}

NS, not significant. 
can be expected when the tumors are completely removed. Klekamp [1] has reported that overall survival (OS) was 92\% for at least 20 years when a GTR is achieved. However, if subtotal resection (STR) of the tumor was performed, the prognosis seems to be worse. According to Oh et al. [2], the 5-year OS after STR was 73.7\%, whereas that after GTR was $98.8 \%$. Therefore, for neurosurgeons, aiming to perform GTR of spinal ependymomas along the tumor-spinal cord border is essential. In contrast, forcible dissection due to inability to perform GTR will lead to neurological sequelae; hence, careful tumor dissection is also important. Particularly, dissection of the ventral aspect of the tumor, which is performed in the last phase of tumor resection, should be paid maximum attention because it can be supplied by the anterior spinal artery [5].

The prognostic factors for spinal ependymomas regarding postoperative neurological status have been reported. Peker et al. [13] reported that TCR was a good indicator of postoperative neurological status, and TCR $>0.80$ was associated with poorer postoperative neurological outcomes. Furthermore, they reported that tumor length was not correlated with postoperative neurological status but was associated with postoperative dysesthesia. Klekamp [1] has reported that preoperative neurological status, thoracic lesions, and presence of tumor hemorrhages were significantly correlated with preoperative morbidities. As with these reports, our results suggested that middle thoracic lesions are associated with unfavorable outcomes in all patients, and high TCRs are correlated with unfavorable outcomes in the single surgery group. However, high TCRs were not correlated with neurological deterioration in the two-stage surgery group; therefore, two-stage surgeries might be beneficial for patients with high TCRs in terms of postoperative outcomes. Moreover, in this study, intraoperative MEP reduction was associated with unfavorable outcomes in all patients and the single surgery group but was not associated with unfavorable outcomes in the two-stage surgery group. These results could be explained by that it would be easy for surgeons to close the first operation immediately after decreasing the MEP when they are willing to perform a two-stage surgery from the beginning, preventing irreversible neurological deterioration.

Previously, several surgeons have performed a twostage surgery for intramedullary spinal tumors. Elsberg and Beer [6] initially reported a two-stage surgery case for spinal gliosarcoma. Benzel et al. [7] and Hida et al. [9] de- scribed two and three cases of spinal astrocytoma treated with two-stage surgery, respectively. Kalangu and Couto [8] reported 20 cases of spinal tumor, including 11 ependymomas, seven astrocytomas, and two schwannomas, which were treated with two-stage resection. This method is neuroprotective because tumors can be spontaneously dissected by the cerebrospinal fluid to some extent and extruded from the spinal cord by intraparenchymal pressure after the first surgery. Moreover, it is an advantage that surgeons can leave the ventral dissection over until the second surgery. Dissection of the ventral aspect of the tumor requires much attention to prevent paresis; however, maintaining focus during the long duration until the final phase, especially when the tumor is large, is difficult. If surgery is continued without concentration, causing an unexpected cord injury will be possible. Moreover, the condition of the spinal cord will improve, owing to decompression after the first surgery. The fragility of the parenchyma is expected to gradually recover as the pressure caused by the tumor has decreased, which could help combat operative stress in the second surgery. Alternatively, two-stage surgery has a disadvantage that patients must feel the burden of the operation twice. Needless to say, finishing tumor resection in one surgery is better if the result will be the same. Therefore, single surgery should be selected in patients with low TCRs.

This study has some limitations. First, it is possible that there were inadequate number of cases. Further cases are expected to accumulate. Second, there is an ununiformity between the two surgical groups. Especially, there was a possibility that patients' age influenced the functional outcome. Therefore, prospective study is required to solve the concern. Third, TCR might be slightly inaccurate because of the low spatial resolution or artifacts of MRI. Because high-spatial-resolution images, such as constructive interference in steady-state MRI or fast imaging employing steady-state acquisition MRI, were not performed in the early cases of our study, the data were obtained using conventional gadolinium-enhanced T1-weighted images. Higher-spatial-resolution images are desired for better evaluation in the future.

\section{Conclusions}

We evaluated the effectiveness of two-stage surgery for spinal ependymomas by retrospectively reviewing our operative series. Our results suggest that 
high TCRs might be a good indication of two-stage surgery and the cutoff TCR is 0.866 . Moreover, switching from single surgery to two-stage surgery may prevent postoperative neurological deterioration when intraoperative MEP is decreasing.

\section{Conflict of Interest}

No potential conflict of interest relevant to this article was reported.

\section{Author Contributions}

Conception and design: Soichiro Takamiya; data acquisition: Soichiro Takamiya, Kazuyoshi Yamazaki; analysis of data: Soichiro Takamiya, Kazuyoshi Yamazaki; drafting of the manuscript: Soichiro Takamiya; critical revision: Toshitaka Seki, Shunsuke Yano, Kazutoshi Hida; and supervision: Toshitaka Seki.

\section{References}

1. Klekamp J. Spinal ependymomas. Part 1: intramedullary ependymomas. Neurosurg Focus 2015;39:E6.

2. Oh MC, Ivan ME, Sun MZ, et al. Adjuvant radiotherapy delays recurrence following subtotal resection of spinal cord ependymomas. Neuro Oncol 2013;15:208-15.

3. Abdullah KG, Lubelski D, Miller J, et al. Progression free survival and functional outcome after surgical resection of intramedullary ependymomas. J Clin Neurosci 2015;22:1933-7.

4. Svoboda N, Bradac O, de Lacy P, Benes V. Intramedullary ependymoma: long-term outcome after surgery. Acta Neurochir (Wien) 2018;160:439-47.
5. Arima H, Naito K, Yamagata T, Kawahara S, Ohata K, Takami T. Quantitative analysis of near-infrared indocyanine green videoangiography for predicting functional outcomes after spinal intramedullary ependymoma resection. Oper Neurosurg (Hagerstown) 2019;17:531-9.

6. Elsberg CA, Beer E. The operability of intramedullary tumors of the spinal cord: a report of two operations with remarks upon the extrusion of the spinal cord. Am J Med Sci 1911;142:636-47.

7. Benzel EC, Mirfarkhraee M, Hadden T, Fowler M. Holocord astrocytoma: a two-staged operative approach. Spine (Phila Pa 1976) 1987;12:746-9.

8. Kalangu KK, Couto MT. Radical resection of intramedullary spinal cord tumors without cavitron ultrasonic aspirator or $\mathrm{CO} 2$ laser: a "two stage" technique. Surg Neurol 1996;46:310-4.

9. Hida K, Iwasaki Y, Seki T, Yano S. Two-stage operation for resection of spinal cord astrocytomas: technical case report of three cases. Neurosurgery 2006;58(4 Suppl 2):ONS-E373.

10. Tobias ME, McGirt MJ, Chaichana KL, et al. Surgical management of long intramedullary spinal cord tumors. Childs Nerv Syst 2008;24:219-23.

11. Yokota H, Yokoyama K, Noguchi H, Uchiyama Y. Two-stage operation for high cervical intramedullary ependymoma in young adult. Br J Neurosurg 2012;26:540-1.

12. McCormick PC, Torres R, Post KD, Stein BM. Intramedullary ependymoma of the spinal cord. J Neurosurg 1990;72:523-32.

13. Peker S, Ozgen S, Ozek MM, Pamir MN. Surgical treatment of intramedullary spinal cord ependymomas: can outcome be predicted by tumor parameters? J Spinal Disord Tech 2004;17:516-21. 